\title{
Brillouin and Raman spectra anomalies in KNSBN with the tungsten bronze structure
}

\author{
Authors: I.G. Siny, S.G. Lushnikov, S.I. Siny, V.H. \\ Schmidt, A.A. Savvinov, and R.S. Katiyar
}

This is an Accepted Manuscript of an article published in Integrated Ferroelectrics in 2000, available online: http://www.tandfonline.com/10.1080/10584580008222223.

I.G. Siny, S.G. Lushnikov, S.I. Siny, V.H. Schmidt, A.A. Savvinov, and R.S. Katiyar, "Brillouin and Raman spectra anomalies in KNSBN with the tungsten bronze structure," Integrated Ferroelectrics 28, 95-102 (2000).

Made available through Montana State University's $\underline{\text { ScholarWorks }}$ scholarworks.montana.edu 


\title{
Brillouin and Raman Spectra Anomalies in KNSBN with the Tungsten Bronze Structure
}

\author{
I.G. SINY ${ }^{a}$, S.G. LUSHNIKOV ${ }^{a}$, S.I. SINY ${ }^{a}$, V.H. SCHMIDT ${ }^{a}$, \\ A.A. SAVVINOV ${ }^{b}$ and R.S. KATIYAR ${ }^{b}$ \\ a Physics Department, Montana State University, Bozeman, Montana, 59717 and \\ b Physics Department, University of Puerto Rico, P.O. Box 23343, San Juan, \\ Puerto Rico 00931-3343
}

(Received May 13, 1999; In final form August 2, 1999)

The Brillouin and Raman scattering from a complex single crystal from the tungsten-bronze family, $\left(\mathrm{K}_{0.5} \mathrm{Na}_{0.5}\right)_{0.2}\left(\mathrm{Sr}_{0.75} \mathrm{Ba}_{0.25}\right)_{0.9} \mathrm{Nb}_{2} \mathrm{O}_{6}$ doped with $\mathrm{Cu}^{2+}$ (KNSBN:Cu), have been comparatively studied in a wide temperature range around the ferroelectric transition. Step-like anomalies in hypersonic velocity and damping confirm the first-order structural transition. These anomalies look like some perturbations on the high-temperature slopes of both a broad dip in sound velocity and a broad maximum in damping that develop in a wide temperature range. The acoustic behavior of KNSBN:Cu does not simply follow the Landau theory prediction valid for many ferroelectrics except relaxors, to which the KNSBN:Cu behavior is intrinsically analogous. The total intensity of the Raman spectra as well as the intensity of separate internal and external vibrations and their width correlate with acoustic anomalies, namely there are step-like drops at the same temperature of the first-order transition and a broad range where the intensity is drastically increased.

Keywords: Brillouin and Raman light scattering; ferroelectric phase transitions; relaxor ferroelectric; tungsten bronze ferroelectric 


\section{INTRODUCTION}

The present work is devoted to the Brillouin and Raman spectroscopy studies of a new single crystal $\left(\mathrm{K}_{0.5} \mathrm{Na}_{0.5}\right)_{0.2}\left(\mathrm{Sr}_{0.75} \mathrm{Ba}_{0.25}\right)_{0.9} \mathrm{Nb}_{2} \mathrm{O}_{6}$ with copper-ion point defects (KNSBN:Cu). This complex compound has been developed as a remarkable photorefractive material [1]. KNSBN originates from the tungstenbronze family of ferroelectrics. KNSBN has two close "descendants" in this family, $\mathrm{Sr}_{x} \mathrm{Ba}_{1-x} \mathrm{Nb}_{2} \mathrm{O}_{6}(\mathrm{SBN})$ and $\mathrm{Ba}_{2} \mathrm{NaNb}_{5} \mathrm{O}_{15}$ (BNN) [2]. $\mathrm{K}$ and $\mathrm{Na}$ additions to SBN increase the Curie temperature of new materials of the KNSBN type. The advantage of large electro-optic coefficients of SBN remains available. The $\mathrm{Cu}^{2+}$ doping provides the further enhancement of the photo-refractive properties of KNSBN [1,3]. From a crystallographic point of view, the KNSBN crystal occupies an intermediate position between SBN and BNN. On the one hand there is a tendency from order to disorder in the sequence of these three compounds. BNN is a mostly ordered material, whereas SBN is rather disordered because $\mathrm{Sr}$ and $\mathrm{Ba}$ ions are distributed randomly and a one-sixth part of interstices remains vacant. On the other hand the crystal structure stability changes in an opposite direction in the sequence. In spite of an obvious disorder, the SBN ferroelectric phase seems to be rather stable below the sole transition, although incommensurate superstructures have been also detected for some $\mathrm{Sr}-\mathrm{Ba}$ compositions [4]. The BNN crystal seems to be very unstable and exhibits a rather unusual sequence of six phases including incommensurate structures [5] (and Refs. therein). Even slight deviations from stoichiometry may produce some discommensurations in BNN.

Thermal stability of the KNSBN structure with the ion arrangement just between that in SBN and BNN is a matter of considerable interest for both applied and scientific approaches. However, the question about the structure stability seems to be open. According to recent Raman scattering studies [6], some hidden transformations may occur in KNSBN below the Curie temperature, manifesting a drastic thermal change in the total intensity of the Raman modes apart from any established phase transition. Analysis of the low-frequency Raman spectra has confirmed $[6,7]$ that the symmetry below $T_{c} \approx 413 \mathrm{~K}$ remains tetragonal in KNSBN:Cu. To our knowledge, no other transitions have been reported for our KNSBN:Cu composition. However, one may suppose that $\mathrm{KNSBN}: \mathrm{Cu}$ should exhibit some phase transition between 60 and $80 \mathrm{~K}$ as has been found in a number of the tungsten-bronze niobate single crystals of three kinds, $\mathrm{SBN}, \mathrm{Pb}_{\mathrm{x}} \mathrm{Ba}_{1 . x} \mathrm{Nb}_{2} \mathrm{O}_{6}$ and $\left(\mathrm{K}_{x} \mathrm{Na}_{1-x}\right)_{0.4}\left(\mathrm{Sr}_{y} \mathrm{Ba}_{1-y}\right)_{0.8} \mathrm{Nb}_{2} \mathrm{O}_{6}$ [8,9]. Combining the Brillouin and Raman spectra analysis, we continue our search for hidden transformations in KNSBN: $\mathrm{Cu}$ from the tungsten-bronze family. 


\section{EXPERIMENTAL}

The KNSBN:Cu single crystal was grown by the Czochralsky method. This crystal was doped with $0.04 \mathrm{wt} \%$ of $\mathrm{CuO}$. Two rectangular samples, one with edges about $5 \mathrm{~mm}$ for Brillouin scattering and another one of $7 \times 5 \times 1 \mathrm{~mm}^{3}$ for Raman scattering, were oriented along the principal axes.

Brillouin backscattering spectra were analyzed by a five-pass Fabry-Perot interferometer. The sample was illuminated along the fourfold axis by an argonion laser with $\lambda=514.5 \mathrm{~nm}$. A narrow-band $(\sim 1 \AA)$ interference filter was used to eliminate the scattering contribution beyond the Brillouin components.

The argon laser with the same wavelength was also used as an excitation source of Raman scattering. Spectra were recorded by a triple grating spectrometer ISA Model T 64000 equipped with a liquid nitrogen cooled CCD detector and with a Raman microprobe system. Both the backscattering and the right-angle geometry were used. For temperature measurements a modified Cryogenic Tech. Closed-cycle Refrigerator Model 20 was used with a LakeShore DRC - 84C temperature controller (accuracy of the temperature control was within about $\pm 0.5 \mathrm{~K}$ ). The raw Raman spectra were deconvoluted using the Jandel Peakfit software program.

\section{Brillouin Scattering}

The longitudinal acoustic phonons, whose frequency was determined by the $c_{33}$ elastic constant, were followed in KNSBN:Cu from room temperature on heating up to $510 \mathrm{~K}$. The Brillouin shift and the width of the Brillouin component at half maximum for the longitudinal acoustic phonons under consideration are shown in Fig. 1 as a function of temperature. The Brillouin shift is proportional to the
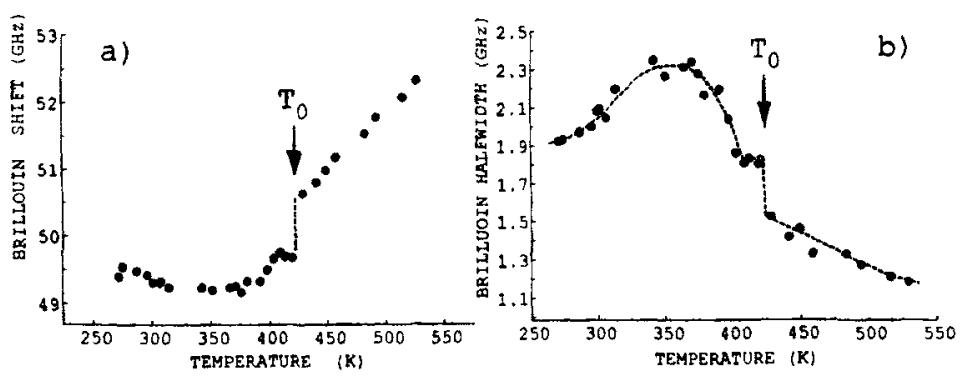

Fig. 1. The temperature dependence of the Brillouin shift (a) and the width at half maximum of Brillouin component (b). The instrumental broadening of 0.8 
hypersonic velocity of phonons whereas the width determines the damping of phonons. As one can see, in the range of the ferroelectric transition, there is a step-like anomaly in the behavior of both the sound velocity and damping. It is well-known that a step-like anomaly for sound velocity is usually expected in the vicinity of a second-order ferroelectric transition if there is a specific connection between the phase transition and elastic properties, namely a coupling squared in order parameter and linear in strain, a $Q \eta^{2} u$-type coupling, where $Q$ is an electrostriction coefficient $[10,11]$. From this point of view, the sound velocity anomaly in KNSBN:Cu resembles the elastic behavior of many other materials with ferroelectric transitions $[12,13]$. However, the hypersonic damping in KNSBN:Cu exhibits a more complex behavior than the Landau-Khalatnikov maximum, which is expected in the case of $Q \eta^{2} \mathrm{u}$-type coupling. The experimental points in Fig. $1 \mathrm{~b}$ show a small "sharp" maximum in damping as the phase transition is approached from below. Then this maximum is followed by a step-like decrease in damping by about twice. On the whole, the anomalous step-like behavior of both the sound velocity and damping rather confirms the first-order phase transition in KNSBN:Cu.

More important and interesting result is how these two elastic characteristics, the sound velocity and damping, are changing in a wide temperature range from 300 up to $500 \mathrm{~K}$. Let us consider their behavior on cooling. The linear reduction in sound velocity in the paraelectric phase is followed by a broad dip in the ferroelectric phase. The hypersonic damping develops like a very broad peak with the maximum value at about $360 \mathrm{~K}$, which is far below the phase transition. Roughly speaking, the velocity dip and damping peak look like reflections of each other in a horizontal mirror plane. "Sharp" anomalies in both the sound velocity and damping in the vicinity of the phase transition appear as a slight perturbation on the high-temperature slopes of the broad dip and of the broad peak respectively.

On the whole, the broad anomalies in sound velocity and damping are very similar to those in some relaxor ferroelectrics from the complex perovskite family, such as PMN and NBT [14-17]. The "sharp" structure of the hypersonic damping in KNSBN:Cu in the vicinity of the phase transition correlates with the "thin" structure of corresponding broad damping maxima in $\mathrm{PbMg}_{1 / 3} \mathrm{Nb}_{2 / 3} \mathrm{O}_{3}$ (PMN) and $\mathrm{Na}_{1 / 2} \mathrm{Bi}_{1 / 2} \mathrm{TiO}_{3}(\mathrm{NBT})$. This similarity confirms a special character of some intermediate phase marked by broad elastic anomalies. Now let us consider what anomalies appear in the Raman spectra of $\mathrm{KNSBN}: \mathrm{Cu}$ in the same temperature range.

\section{Raman Scattering}

The single crystal of KNSBN:Cu shows a set of differently polarized spectra with many lines that are rather broad and overlap one another. The diagonal ( $Z Z)$ 
Raman spectra taken in the backscattering geometry are depicted in Fig. 2 at five

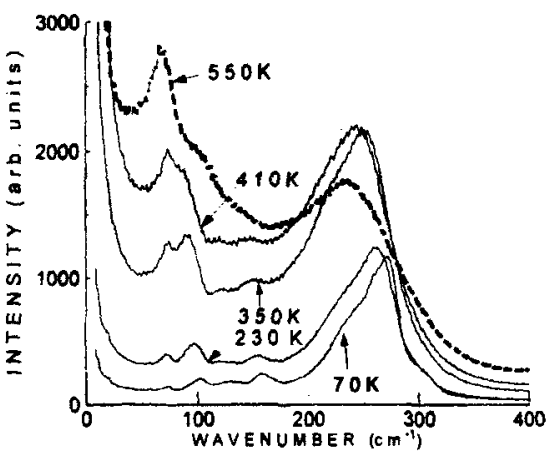

Fig. 2. Thermal evolution of the (ZZ) Raman spectra. The raw data are presented. characteristic temperatures. The diagonal $(Z Z)$ spectrum is the most intense one among all polarized spectra. The $(Z Z)$ spectrum differs considerably from two other diagonal spectra $(X X)$ and $(Y Y)$ which are almost identical $[6,7]$. This important difference implies a tetragonal symmetry at room temperature in accordance with the character of the Raman tensor, namely only the $A_{1}(z)$ modes appear in the $(Z Z)$ spectrum whereas both the $A_{i}(z)$ and $B_{1}$ modes are present in the $(X X)$ and $(Y Y)$ spectra. No difference among three diagonal spectra appeared in the main ferroelectric phase of BNN around room temperature, and that result was considered as a proof of the orthorhombic structure $[5,18]$.

The difference between the diagonal spectra is clearly seen in the lowfrequency part of the Raman spectra, $v>120 \mathrm{~cm}^{-1}$, which consists of external lattice vibrations involving cation motions relatively to the oxygen octahedron framework (Fig. 2). The framework vibrations are represented by two sets of strong Raman lines of the $A_{1}(z)$ symmetry around 260 and $640 \mathrm{~cm}^{-1}$ (Fig. 2). These groups of intemal modes are characteristic of the tungsten-bronze structure $[18,19]$. The external mode analysis $[6,7]$ evidences that the KNSBN:Cu crystal has the tetragonal structure characteristic of the tungsten-bronze type ferroelectrics in spite of a formal similarity to BNN. The tetragonal SBN crystal exhibits spectra similar to that in KNSBN:Cu although the low-frequency $\mathrm{B}_{1}$ mode has not been found [19].

However, the tetragonal character of the Raman spectra in KNSBN:Cu does not exclude the possibility of incommensurate structures. The orthorhombic symmetry of BNN manifests itself by some characteristic details in the Raman spectra, namely there are only $A_{1}(z)$ modes in the three diagonal spectra and the initial $E$ modes from the tetragonal high-temperature phase appear to be split into pairs of $B_{1}$ and $B_{2}$ symmetry in the off-diagonal $(X Z)$ and $(Y Z)$ spectra. These characteristic features remain pronounced in both adjacent incommensurate phases at lower and higher temperatures [5]. The characteristic splitting vanishes only above the incommensurate-commensurate transition to the real tetragonal phase [5]. 
Soft modes have been found by Raman scattering neither in KNSBN:Cu of the present composition nor in other close KNSBN crystals [20]. The most pronounced anomaly in the Raman spectrum behavior of $\mathrm{KNSBN}: \mathrm{Cu}$ at the ferroelectric phase transition could be connected with a change in the selection rules when the ferroelectric $4 \mathrm{~mm}$ point group transforms into the $4 / \mathrm{mmm}$ point group with the inversion center. Only about half as many modes remain Ramanactive in the high-temperature phase whereas the other modes are only infraredactive. This transformation implies that the total spectrum intensity is a sensitive tool to detect the ferroelectric phase transition. Comparison of the (ZZ) spectra at different temperatures (Fig. 2) shows that in fact the intensity of the broad band around $260 \mathrm{~cm}^{-1}$ changes drastically in the range of the transition. Moreover, this band obviously continues changing in intensity even at lower temperatures in the ferroelectric phase.

In order to obtain a correct result, we eliminated from our consideration the trivial temperature dependence of the mode intensity due to the population factor. The thermal behavior of the scattering cross-section in KNSBN:Cu was calculated in a wide temperature interval from 70 up to $580 \mathrm{~K}$. The spectral intensity was integrated in the range from 20 to $400 \mathrm{~cm}^{-1}$ that included the most characteristic Raman spectrum of the tungsten-bronze structure, namely several external lattice modes as well as the strong internal bending modes of the $\mathrm{NbO}_{6}$ octahedron. The intensity integration was restricted to $400 \mathrm{~cm}^{-1}$ because there are no Raman lines in the range from 350 to $550 \mathrm{~cm}^{-1}$ (Fig. 2). The lower curve (a)

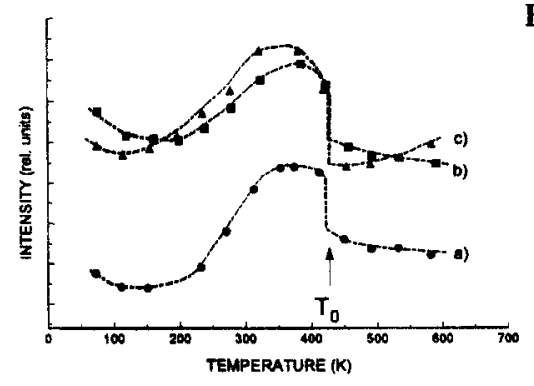

Fig. 3. The temperature dependence of mode intensity in the (ZZ) Raman spectra: (a) integrated intensity (integration in the range from 20 to $\left.400 \mathrm{~cm}^{-1}\right)$; (b) - an internal vibration at around $260 \mathrm{~cm}^{-1}$ (O-Nb-O bending); (c) - an external vibration at about $120 \mathrm{~cm}^{-1}$

in Fig. 3 shows that there is an abrupt reduction in intensity at about $420 \mathrm{~K}$, which could be expected, and in addition there are two unexpected anomalies in the ferroelectric phase. There is a drastic change in intensity in the range $200-$ $350 \mathrm{~K}$ and an anomalous reduction in intensity above a possible additional transition at about $70 \mathrm{~K}$ that has been found in the related KNSBN materials $[8,9]$. One should emphasize that the intensity change between 200 and $350 \mathrm{~K}$ exceeds the anomalous drop at about $420 \mathrm{~K}$ although no transition has been 
reported in that temperature region for the KNSBN family. Thus, the Raman scattering studies provide new evidence that the structure of KNSBN:Cu in the ferroelectric phase is not very stable.

\section{CONCLUSIONS}

The complex evolution of KNSBN:Cu to the ferroelectric state has been revealed by light scattering experiments. No soft mode has been found in Raman scattering. However, other characteristics, such as mode intensity, indicate the complex dynamic evolution that includes the first-order structural transition followed by a broad anomalous phase. The behavior of the longitudinal acoustic phonons in Brillouin scattering does not show any normal coupling with a possible but "unseen" soft mode. Their behavior confirms the first-order transition and reveals an anomalous phase below the first-order transition, in agreement with Raman scattering.

We can assume that an incommensurate structure in $\mathrm{KNSBN}: \mathrm{Cu}$ is quite possible, especially taking into account some energy competition between the commensurate and incommensurate structures in the thermal evolution [21]. Further structural studies are needed.

\section{ACKNOWLEDGMENTS}

We thank H.-C.Chen for providing the samples. The work was supported in parts by DAAG-55-98-1-0012, NSF DMR-9801759 and NSF DMR-9805272 Grants and RFBR Grant No. 99-02-18316.

\section{References}

[1] Q. Jiang, X. Lu, Y. Song, D. Sun, and H. Chen, Phys. Rev. B 50, 4185 (1994).

[2] M.E. Lines and A.M. Glass, Principles and Applications of Ferroelectrics and Related Materials (Clarendon, Oxford, 1977).

[3] X. Yue, X. Lu, Y. Song, Z. Shao, D. Sun, Q. Jiang, and H. Chen, Appl. Phys. 53, 319 (1991).

[4] L.A. Bursill and P.J. Lin, Philos. Mag. B 54, 157 (1986); Acta Cryst. Sect. B 43, 49 (1987).

[5] A. Shawabkeh and J.F. Scott, Phys. Rev. B 43, 10999 (1991).

[6] A.A. Savvinov, I.G. Siny, R.S. Katiyar, and H.-C. Chen, J. Korean Phys. Soc. 32, S617 (1998).

[7] A.A. Savvinov, I.G. Siny, R.S. Katiyar, and H.-C. Chen, Proc. XV-th Intern. Conf. on Raman Spectroscopy, edited by S.A. Asher (John Wiley \& Sons, 1966), p. 878.

[8] Y. Xu, Z. Li, W. Li, H. Wang, and H. Chen, Phys. Rev. B 40, 11902 (1989).

[9] Y. Xu, Z. Li, W. Li, H. Wang, and H. Chen, Ferroelectrics 108, 253 (1990).

[10] J.C. Slonczewski and H. Thomas, Phys. Rev. B 1, 3599 (1970).

[11] W. Rehwald, Adv. Phys. 22, 721 (1973).

[12] B. Luthi and W. Rehwald, in Structural Phase Transition I, edited by K.A. Müller and H. Thomas, Topics in Current Physics, Vol. 23, (Springer, Berlin, 1981), p. 131. 
[13] H.Z. Cummins, in Light Scattering Near Phase Transitions, edited by H.Z. Cummins and A.P. Levanyuk (North-Holland, Amsterdam, 1983), p. 359.

[14] I.G. Siny, C.-S. Tu, and V.H. Schmidt, Phys. Rev. B 51, 5659 (1995).

[15] I.G. Siny, S.G. Lushnikov, C.-S. Tu, and V.H. Schmidt, Ferroelectrics 170, 197 (1995).

[16] I.G. Siny, S.G. Lushnikov, R.S. Katiyar, and V.H. Schmidt, Ferroelectrics 226, 191 (1999).

[17] C.-S. Tu, V.H. Schmidt, and I.G. Siny, J. Appl. Phys. 78, 5665 (1995).

[18] A. Boudou and J. Sapriel, Phys. Rev. B 21, 61 (1980).

[19] R.E. Wilde, J. Raman Spectr. 22, 321 (1991).

[20] T. Chen, X. Shao, Y. Xu, and H. Chen, Ferroelectrics 82, 37 (1988).

[21] B.A. Strukov and A.P. Levanyuk, Ferroelectric Phenomena in Crystals (Springer-Verlag, Berlin, Heidelberg, 1998), p. 257. 